\title{
Genre Cataloging and Instrument Classification in Large Databases using Music Mining Approach
}

\author{
R.Rebekha \\ Assistant Professor, Department of Computer Science \& Engineering \\ Prist University, Thanjavur, Tamilnadu, Country. \\ M.Joseph \\ Professor, Department of Computer Science \& Engineering \\ St.Joseph's College of Engineering \& Technology, Thanjavur, Tamilnadu, Country.
}

\begin{abstract}
Mining of music information has dependably been always a developing area of interest where information's are stored in large stash. A challenge in music information management is how to utilize information indexing systems in view of various aspects of the information itself. For music data, content and different acoustic components can be connected to encourage efficient music management. In Mining of music information, characterization tasks are subdivided into genre cataloging, instrument distinguishing and artist classification. Apart from classification, other information mining tasks are comparable for music applications. In this Paper a collaborative music-mining classifier (CMM-C) system which is based on self-adaptive harmony search algorithm. In the pre-processing stage, fundamental acoustic qualities are separated and stored as original feature set. CMC is created with altered wavelet transformation (for processing acoustic signals). Conventional frameworks utilize Fourier transform for the classifications, but from the survey it is observed that remaking high frequency coefficients are not possible from the modulus complex convolution. The classification through wavelet infuses mining instantaneous frequency of music information, mounting highlights with sub-band coefficients and spatial-temporal energy location curve. To progress the accuracy in classification to take high incidence coefficients into the processing and by solving the modernization issue with altered wavelet transform to organize the genre with higher achievement rate. As the result of evaluation it is able to complete the genre cataloging and instrument characterization with CMM classifier. The approval of this mining procedure is reasonably compared with other harmony search algorithms, multi-layer perceptrons and SVM techniques.
\end{abstract}

Keywords - Harmony Search Algorithm, CMM-C (Collaborative Music- Mining Classifier), Wavelet Transformation, Mel-Frequency Cepstral Coefficients (MFCC), Music Genre and Instrumental Classification.

\section{INTRODUCTION}

As the measure of accessible music-related data expands, the difficulties of arranging and investigating such information become paramount. As of late, numerous data mining methods have been utilize to perform different tasks (e.g., genre classification, emotion and mood detection, playlist generation, and music information retrieval) on music-related information sources. Data mining is a procedure of automatic extraction of novel, helpful and justifiable patterns from a substantial accumulation of information. With the substantial amount of accessible data from different sources, music has been a general application area for data mining methods. Audio information is an integral part of many modern computer and multimedia applications. A typical multimedia database regularly contains millions of audio clips, including environmental sounds, machine noise, music, animal sounds, speech sounds, and other non-speech utterances. Analysts extract music score for further discussion on music computable. The adequacy of their deployment is greatly dependent on the ability to characterize and recover the sound documents as far as their sound properties or substance. The need to consequently perceive to which class an audio sound belongs makes sound characterization and categorization an emerging and important research area.

In this paper, programmed sound component extraction and grouping methodologies are displayed. In order to segregate the six categories of audio namely music, news, sports, advertisement, cartoon and movie, a number of features such as LPC, LPCC, MFCC are separated to classify the sound substance. The five layer auto associative neural network model as portrayed in Section 3.1 is utilized to capture the distribution of the facial element vectors. The AANN model is utilized for capturing the distribution of the acoustic components of a class, and the back propagation learning algorithm is utilized to modify the weights of the network to minimize the mean square error for each element vector. Experimental results demonstrate that the classification accuracy of GMM with Mel- 
cepstral elements can give a better result. Figure 1 shows the block diagram of audio classification. A hybrid model contained Gaussian mixtures models (GMMs) and hidden Markov models (HMMs) is utilized to model generic sounds with expansive intra class perceptual variations [1].

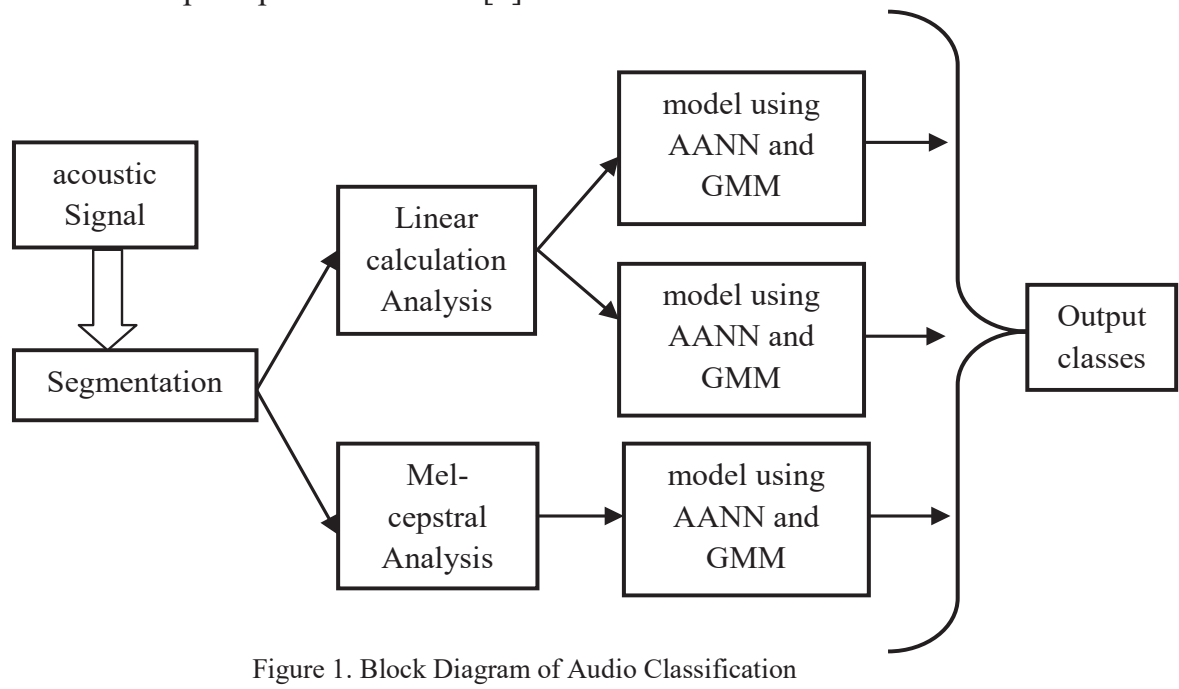

The spectral data of the same sound expressed by two persons might contrast because of change in the shape of the individual's vocal tract system, and the way of discourse creation. The selected elements include linear prediction coefficients (LPC), linear prediction derived cepstrum coefficients (LPCC) and mel-frequency cepstral coefficients (MFCC). Mel-frequency cepstral coefficients are short term spectral based elements utilized by numerous researchers for speech recognition [3], recovery framework [4], music summarization [5], speech/music discrimination [6]. The quality of MFCC is in compact representation of amplitude spectrum. The progressions for computing MFCC as explained in [7] have been shown in Figure 2.

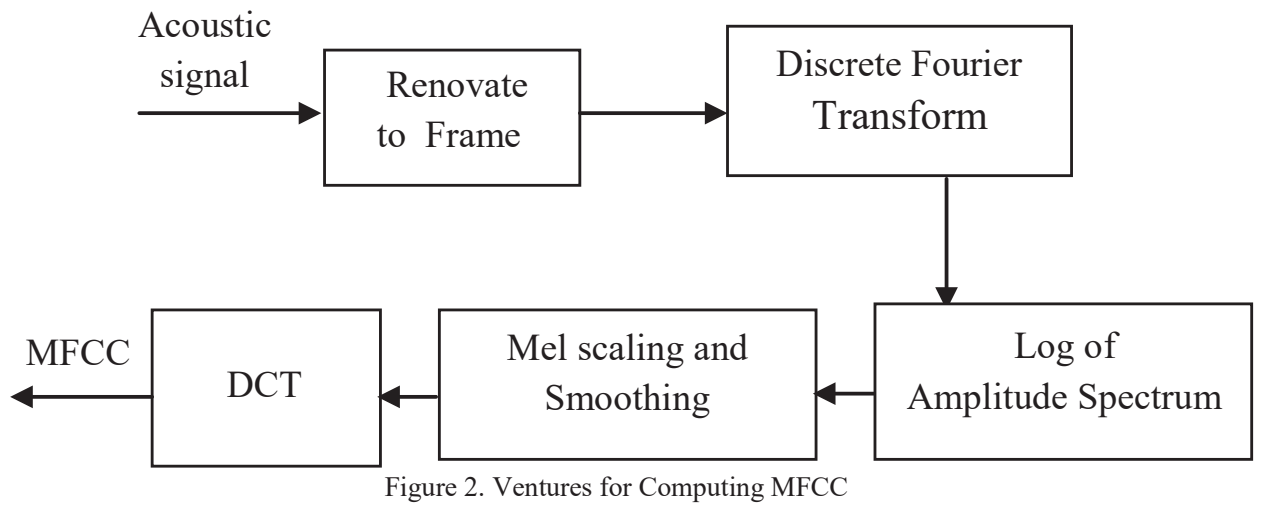

Psycho-accoustic Music Descriptors as proposed by [2] depend on a psycho-acoustically changed Sonogram representation that reflects human loudness sensation. Statistical Spectrum Descriptors (SSD) consequently computes statistical moments for the 24 critical bands of hearing. Rhythm Patterns (RP) describe fluctuations in modulation frequency which give a rough interpretation of the rhythmic energy of a song. Rhythm Histograms (RH) aggregates the modulation amplitude estimations of the individual critical bands registered in a RP. Temporal Variants (TSSD, TRH) describe variations over time through statistical moments calculated from consecutive segments of a track. The music signal, be it instrumental or song, might have a broad variety making the task of classification critical. In this connection a direct threshold based methodology is quite prohibitive. Therefore, numerous researchers have relied on the classification plans like neural network, SVM. The variation even within a class of audio signal might give rise to outliers putting detrimental bias in classification. Audio signal is initially partitioned into number of frames of fixed duration. Frames may consist of samples with an overlap with the previous frame. To minimize the irregularity at the beginning and end of the frame a windowing function (Hamming window is the most broadly utilized one) is additionally connected on the frame. Amplitude spectrum for each (windowed) frame is obtained by applying Discrete Fourier Transform (DFT). 


\section{RELATED WORKS}

Music assumes an important role in the regular life for some individuals, and with the digitalization, vast music information collections are framed and tend to be gathered further by music enthusiasts. A framework that naturally organizes a music accumulation as indicated by the apparent sound similarity resembling genres or styles of music. In their methodology, audio signals are handled according to psychoacoustic models to acquire a time-invariant representation of its qualities. Subsequent clustering gives an intuitive interface where comparable pieces of music are gathered together on a map display. Give some related definitions and present the issues in directory ontology matching. Figure 3 shows the directory ontology, the "Music" idea has two names "Music" and "Mus.". So, the local label of this idea is \{Music,Mus.\} and the way mark of "Music" is \{Entertainment\}/\{ Music,Mus. \} So far, for every idea $c$ in directory ontologies and get two kinds of data of it: $L(c)$ and $P L(c)$.
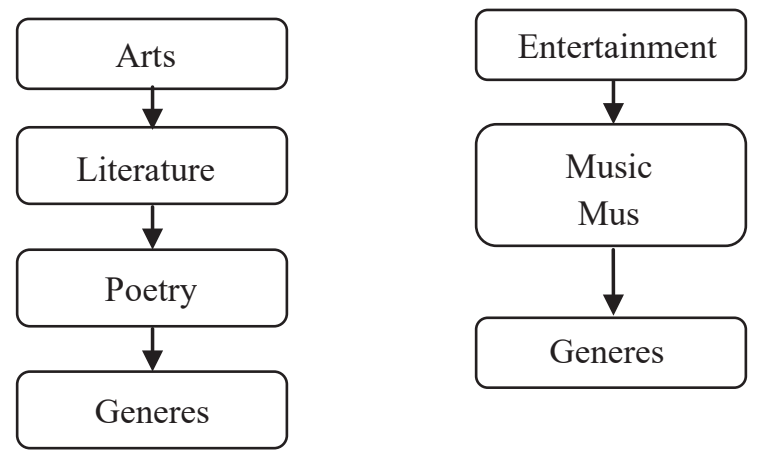

Figure 3. Same labels but distinctive significance

In Figure 3, in spite of the fact that the leaf labels are propositional (namely Genres), they represent various meaning. The idea of "Genres" on the left path indicates a poetry genres and the idea"Genres" on the right means a music genres. The subject of computationally perceiving these attributes of music, where parts of the work exhibited in this paper have showed in [10], [11], [12], [13], [14]. The four types of qualities are strongly related to each other.

\section{A. Watermark Mel-Frequency Cepstral Coefficients (MFCC)}

MFCC is a list of capabilities prevalent in speech processing and music modelling [8]. This element is acquired as follows: First compute, for every frame, the logarithm of the amplitude spectrum based on short-term Fourier transform, where the frequencies are partitioned into thirteen bins utilizing the Mel-frequency scaling. (The "cepstrum" is the FFT of this logarithm.) Then apply discrete cosine transform to de-correlate the Mel-spectral vectors. In this study, utilizing the first five bins, and compute the mean and variance of each over the frames.

\section{B. $\quad$ Short-Term Fourier Transform Features (FFT)}

This is an arrangement of components identified with timbral textures and is not captured utilizing MFCC. It comprises of the accompanying five types. More detailed portrayals can be found in [9]. Spectral Centroid is the centroid of the amplitude spectrum of short-term Fourier transform and it is a measure of spectral brightness. Formally, the spectral centroid $\boldsymbol{P t}_{\boldsymbol{t}}$ is characterized as

$$
P_{t}=\frac{\sum_{n-1}^{N} X_{t}[n] * y}{\sum_{n-1}^{N} X_{t}[n]}
$$

Where is the magnitude of the Fourier transform at frame $t$ and frequency bin $y$.

Zero Crossings is the quantity of time domain zero crossings of the signal. It measures the noisiness of the signal. Formally, the time domain zero crossings is characterized as,

$$
{ }_{z t=\frac{1}{2}} \sum_{n=1}^{N}|\operatorname{sgn}(x[n])-\operatorname{sgn}(x-[n-1])|
$$

where $\mathrm{x}[\mathrm{n}]$ is the time domain signal for frame $\mathrm{t}$ and $\mathrm{sgn}$ is the sign function (e.g., it takes the estimation of 1 for positive arguments and 0 generally.) Compute the mean for every one of the five and the variance for all but zero 
crossings. The personality of a sound is mostly affected by the magnitude spectrum in spite of fact that the phase assumes an essential role particularly near transient parts of the signal such as percussion hits. The utilization of bidimensional visualization techniques to music collections has to be carried out considering that the visualization will be given to non-expert clients, as opposed to information analysts, who require a simple and appealing representation of the data. Generally, constructing models when one class is uncommon is quite difficult because there are often many unstated assumptions [20]. A conventional wisdom is that classifiers built utilizing all the information tend to perform worse on the minority class than on the majority class since the class priors in the natural distribution are biased strongly in favor of the majority class and the minority class has much fewer training and test samples [21].

\section{PROPOSED METHOD}

A more modern administration of computerized music documents can be done with special software or with augumented music players, like Winamp or Windows Media Player. For this situation, the included meta information in music documents is utilized for the association and this methodology enables an efficient keywordbased search mechanism for music files. Additionally this methodology works well for small music file accumulations, the utilization of a connection database system is crucial for substantial music collections. The benefit of database systems is that they provide functionalities to deal with large data sets as well as efficiently search methods within the information sets. The sample Music tones are named S1,S2,S3,S4,S5 and S6 is shown in Figure 4.

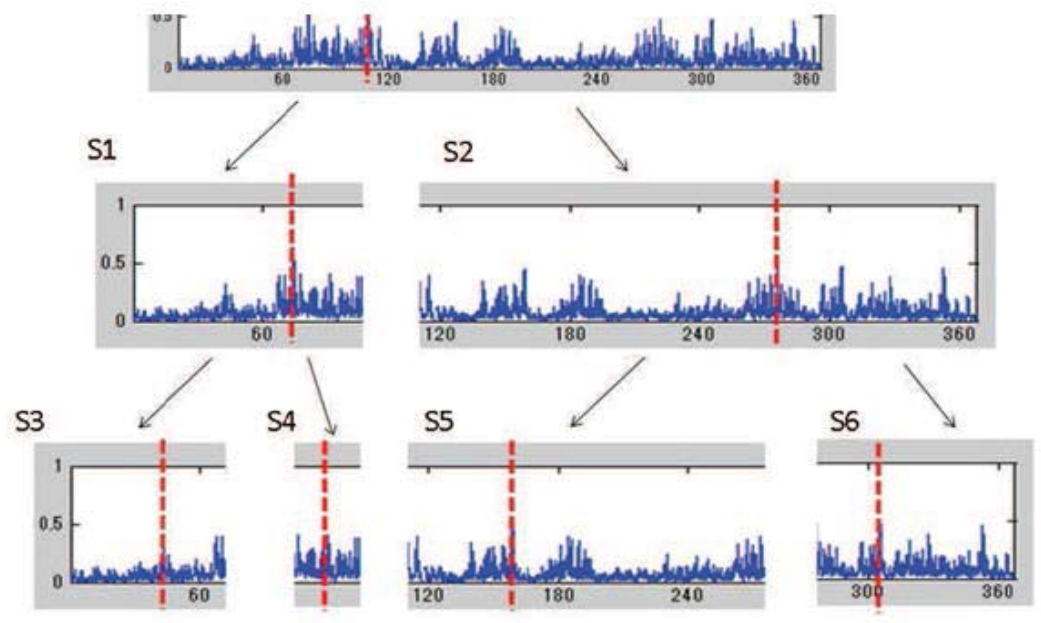

Figure 4. Same Music tones for database

The representation of the song by components to be utilized by the learning algorithm is also a critical aspect of the system. To the extent music is concerned, these features can be separated not only from the audio recordings but also from a variety of information sources surrounding the musical substance, in particular different types of metadata normally connected to it, particularly cover art and lyrics (in sung music). In our method, we combined Genere cataloging and instrument classification by using space reduction of the database. Generally, many researchers are designed separate algorithm for every label, then used supplementary space in particular framework. But recently our combination work is mentioned the overall algorithm is premeditated in the single database. For that reason, the space is concentrated in our technique and we avoid the corruption of music data.

\section{A. A Collaborative Music-Mining Classifier System}

In this segment, a new grouping system known as a collaborative multi-aspect classifier (CMAC) is presented. This framework utilizes a set of collaborating agents that communicate with each other prior to making a final decision. The improvement of the CMAC framework is inspired by its collaborative capacity to minimize a cost function based on overall misclassifications. Each agent imparts this information with other agents in the group utilizing a coordinator, whose capacity is to encourage the sorting and transmission of the appropriate preliminary decisions to and from every agent. Upon the receipt of $u_{i} s i=1, \ldots \ldots, N$, the coordinator makes $N$ vectors denoted $u_{i r}$, $\mathrm{i} \in[1, \mathrm{n}]$, where 


$$
u_{i r}=\left[u_{1}, \ldots, u_{i-1}, u_{i+1}, \ldots u_{N}\right]^{T}
$$

Note that the list term ir is utilized to denote that this vector will be received by the $i^{\text {th }}$ agent for use in its final decision computation. Once these vectors are shaped, the coordinator subsequently transmits each to the appropriate agent's BPNN probability estimator (shown in Figure 5), whose capacity is to appraise the class conditional probability terms required for ascertaining the threshold utilized in the final decision by each agent. the proposed CMAC framework (shown in Figure 6), a group of $\mathrm{N}$ decision-making agents is utilized to create $\mathrm{N}$ separate decisions regarding $\mathrm{N}$ pattern vectors (observations) $\mathrm{x}_{i}, \mathrm{i}=1, \ldots \mathrm{N}$. This is accomplished in the following manner. First, an agent $i$, whose internal structure is shown in Figure 5, create a preliminary decision, assigned by $u_{i}$, using a two class probabilistic neural network (PNN) based on the pattern available to the agent. The reason of this preliminary decision is to assist the other $\mathrm{N}-1$ agents in making their final decisions. These preliminary decisions are chosen to be continuous-valued scalars whose values vary between zero and one, and are intended to speak the confidence of an agent regarding the class of the element vector.

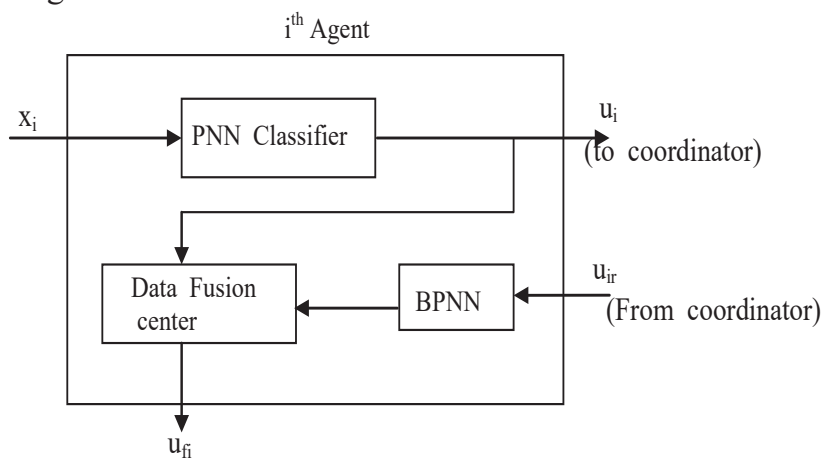

Figure 5. Internal structure of the $i$ th agent

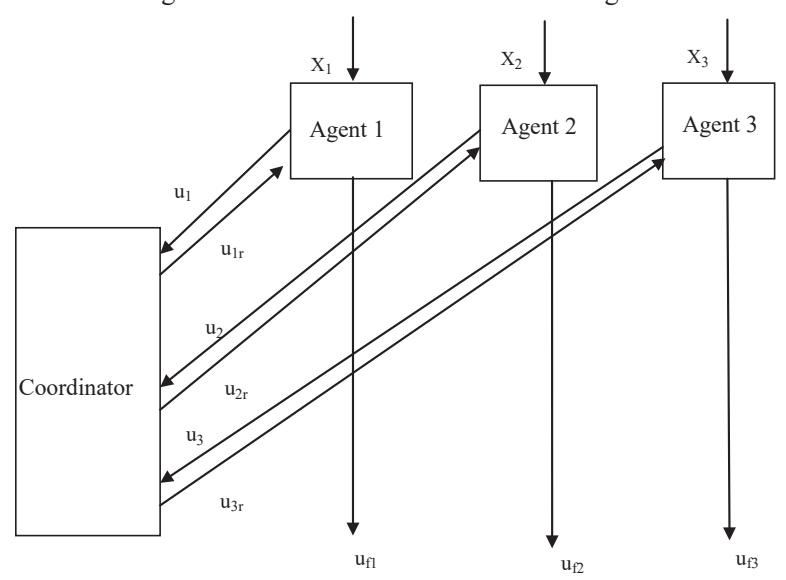

Figure 6. Representation of CMM Classifier

\section{B. Discrete wavelet Transform based Music samples}

The feature set for representing rhythm structure is depends on indentifying the most salient periodicities of the signal. Figure 7 demonstrates the flow diagram of the beat analysis algorithm. The signal is first decomposed into a number of octave frequency bands utilizing the DWT. Taking after this deterioration, the time domain amplitude envelope of every band is removed independently. This is accomplished by applying full-wave rectification, low pass filtering, and downsampling to each octave frequency band. After mean removal, the envelopes of each band are then summed together and the autocorrelation of the resulting sum envelope is figured. The dominant peaks of the autocorrelation capacity compare to the different periodicities of the signal's envelope. These peaks are collected over the entire sound file into a beat histogram where each bin corresponds to the peak lag, i.e., the beat period in beats-per-minute (bpm). Instead of including one, the amplitude of each peak is added to the beat histogram. That way, when the signal is fundamentally the same to itself (strong beat) the histogram peaks will be higher. 
Discrete Wavelet Transform Octave Frequency Bands

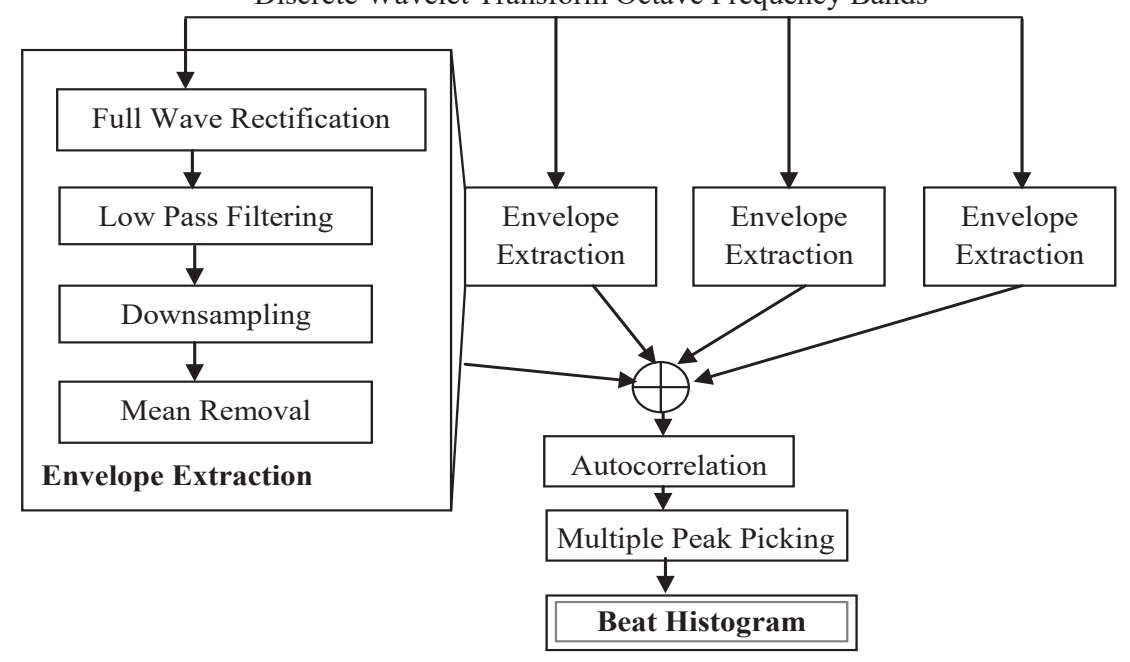

Figure 7. Beat histogram estimation of wavelet transform frequency bands

\section{Architecture of Tones in Music-mining Process}

The architecture of T-Music is shown in Figure 8. In Phase I, given a song database, containing a lot of existing songs, and a tone look-up table, containing the mapping between the syllable of each word and the tone ID. For each song in the song database, T-Music performs the Tone Extraction utility on its lyric, and obtains the tone sequence and thus the s-sequence. At that point, it mines every regular patterns based on all s-sequences found.

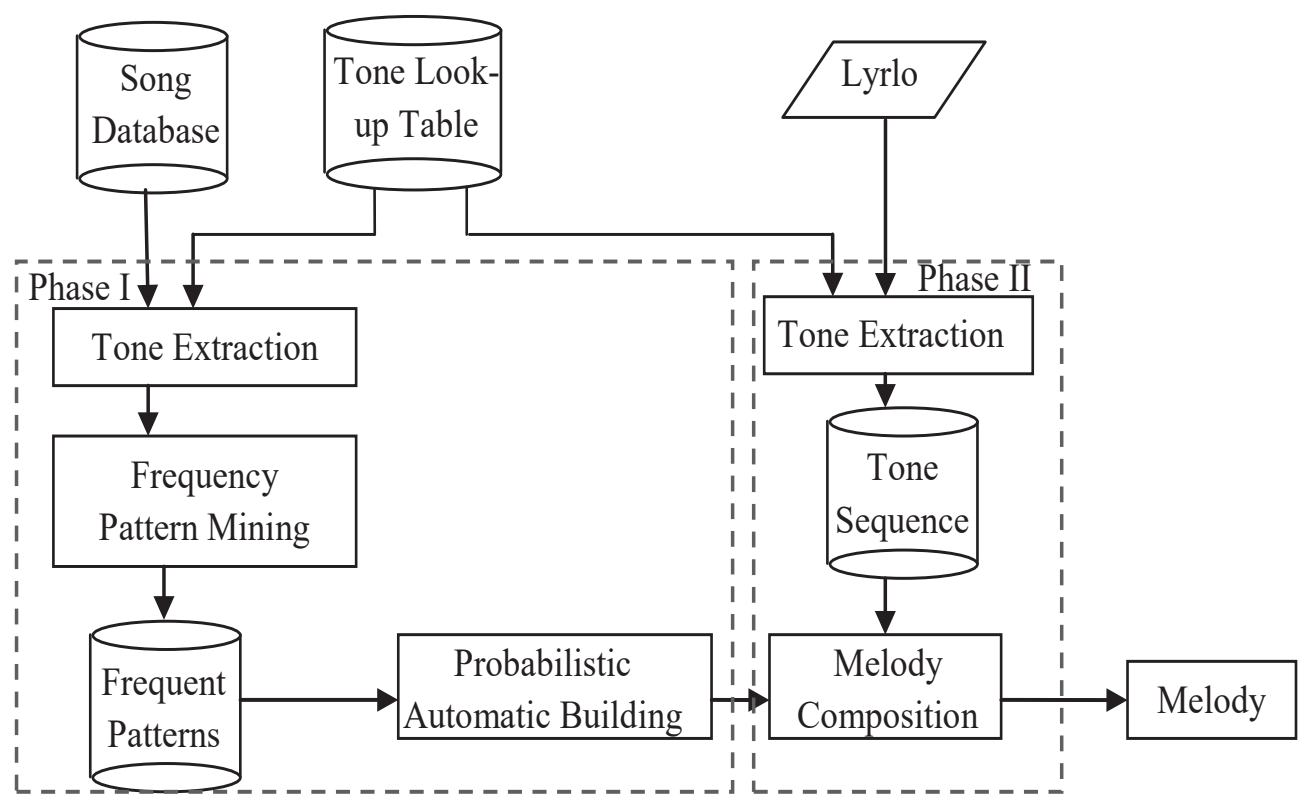

Figure 8. Architecture of tones utilizing Music mining

Here, the frequent pattern compares to the lyric-note correlation. At that point, it fabricates a Probabilistic Automaton (PA) based on these frequent patterns. In Phase II, the same tone look-up table and an input lyric written in a language are given. T-Music separates the tone sequence from the input lyric via a Tone Extraction utility. Then, it performs the Melody Composition process by executing the constructed PA with the input of the removed tone arrangement, which outputs a melody. In the accompanying Section II-C, will present three key utilities of TMusic, namely Frequent Pattern Mining, Probabilistic Automaton Building and Melody Composition, in detail. 


\section{Self Adaptive Harmony Search Algorithm}

The metaheuristic harmony search (HS) algorithm is a population-based developmental algorithm that is prpolled by the improvisation process of musicians when making a new piece of music. The HS algorithm has increased widespread attention among researchers from various disciplines. This music-inspired algorithm has been produced and utilized to solve a wide range of optimization issues in the field of science and designing. The success of this HS algorithm stems from the fact that this metaheuristic methodology offers numerous attractive advantages.

\begin{tabular}{ll}
\hline \multicolumn{1}{c}{ Algorithm } \\
\hline Step 1 & Initialize parameters \\
Step 2 & Initialize harmony memory (HM) \\
Step 3 & Improvise a new harmony (solution) \\
Step 4 & Update HM if a better solution is found \\
Step 5: & Repeat step 3 and 4 until stopping criterion is \\
& met \\
\hline
\end{tabular}

Figure 9. Procedure of Harmony Search Algorithm (HS)

To utilize the HS algorithm in the issue of feature selection, two different methodologies can be figured. The two new methodologies, specifically horizontal methodology and vertical methodology, are introduced. In horizontal approach, each musician is assigned one of the two binary values. On the contrary, for vertical approach, a different strategy is utilized. Here, every musician is treated as an individual expert who will identify which element to be chosen and included in the feature subset. Different musicians are permitted to pick the same element. Moreover, they can pick no component at all. The final harmony is created based on the decisions made by all the individual musicians. The merit of the vertical approach is that it makes good use of the principles embedded in the HS algorithm, where a diverse range of applicant arrangements can be created. All things considered, the drawback of the methodology is that an extra tunable parameter should be presented, which complicates the structure of the algorithm.

\section{Improvise a new harmony}

Improvise a new harmony During this step, a new harmony, or a new applicant arrangement, is improvised based on the existing arrangements stored in HM. The new estimation of a decision variable, is acquired utilizing the accompanying rules,

$$
X_{i}^{\prime}=\left\{\begin{array}{cc}
X_{i} \in\left[X_{i}^{L}, X_{i}^{U}\right] & \text { ifr }>\text { HMCR } \\
x_{i} \in H M=\left\{X_{i}^{1}, X_{i}^{2}, X_{i}^{3}, \ldots, X_{i}^{H M S}\right\}, & \text { ifr }<H M C R \quad i=1,2, . ., N . \\
X_{i}+\alpha\left(X_{i}^{L}{ }_{-} X_{i}^{U}\right) \mid X_{i}^{\prime} \in H M & \text { ifr }<P A R,
\end{array}\right.
$$

\section{2. $\quad$ Update Harmony memory}

Update harmony memory For each of the recently created arrangement in Step 3, its quality will be assessed utilizing the cost capacity, $f$. The output, $f\left(X_{i}^{\prime}\right)$, is contrasted with all the other outputs stored in HM. If the new arrangement is observed to be better, the new arrangement vector, $X_{i}^{\prime}$ will replace the worst solution in HM. Numerically, the rule is given as:

$$
X^{\prime} \in H M \wedge X^{\text {worst }} \notin H M
$$

An algorithm called self versatile harmony search (SHS) algorithm is given, where the BW parameter is supplanted and the new harmony is upgraded according to the maximal and minimal values stored in HM. The two formulas proposed are as per the way that the maximum and minimum values of variables would approach their respective optimum values progressively. The pitch conformity principle proposed through this mechanism does not violate the boundary constraints imposed on the decision variables. Besides, an initialization strategy based on the concept of low-discrepancy sequences is utilized instead of the standard pseudo- random number based approach. The support given being that the values created by the low-discrepancy sequences are more equitably appropriated. 


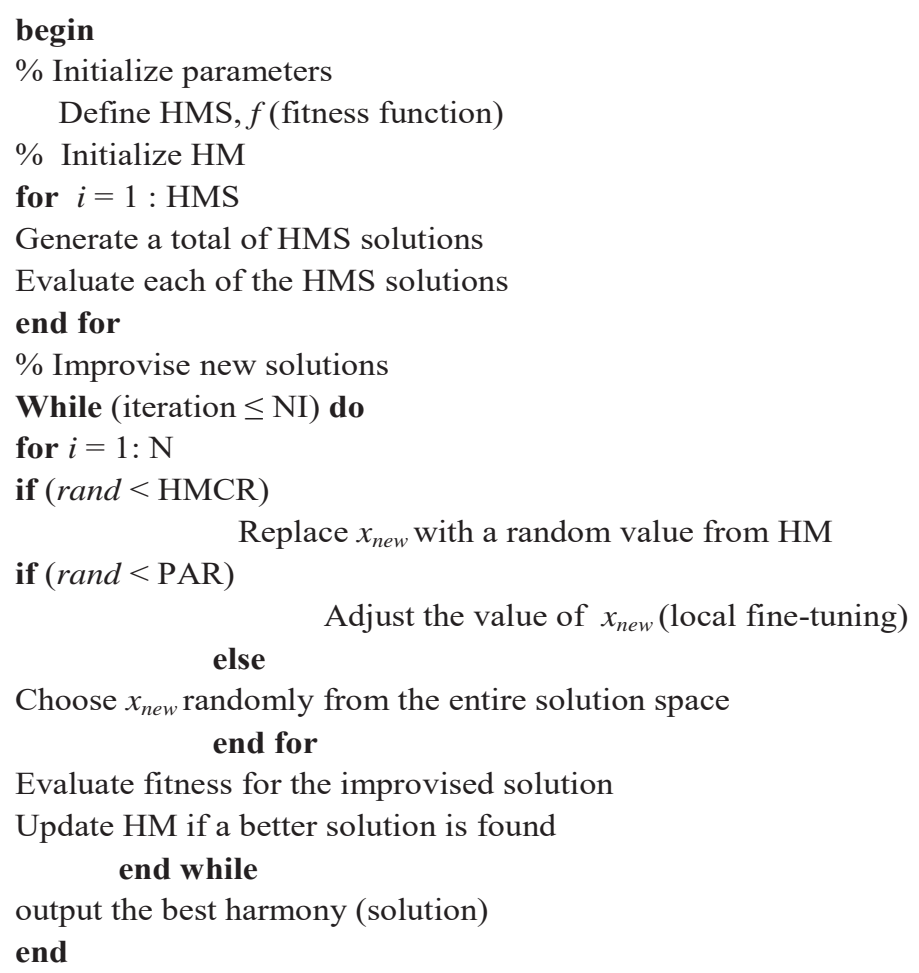

Figure 10. Pseudo code of the standard harmony search algorithm

\section{PRoposed COLLABORATIVE MUSIC-MINING CLASS}

A. Pre-Processing Algorithm for (CMM-C)

Wave_class (Data, $\mathrm{SV}_{\min }, \mathrm{CV}_{\min }$, $\operatorname{minG}, \operatorname{maxG}$ )

Initialize

$\mathrm{D}^{\prime}=$ construct Wave (D);

$\mathrm{Q}\{\}=$ Ruleform (D' $\left.\mathrm{SV}_{\min }, \mathrm{CV}_{\min }, \operatorname{maxG}\right)$

Function construct wave (D)

begin

$\mathrm{D}^{\prime}=$ null set;

Count $\left(\right.$ Wave $\left._{\text {coeff }}\right)=1$. String size $_{\text {s. }}$

$\lambda_{n}^{i}(j)=\left(\varnothing_{n+1}^{2, i-1}(j)-\varnothing_{n+1}^{2, i}(j)\right) / 2$

$\lambda_{n}^{i}(j) \leftarrow \lambda_{n+1}^{i+1}(j+1),(l / 2)$

Update D' with new

End

B. Classification Algorithm for (CMM-C)

Rule form (D' SVmin, CVmin, maxG)

Initialize Rule $_{\text {count }}$, Rule pointer $_{\text {. }}$

$\Delta_{x}=U_{0}^{\max G} \mathcal{W}_{x}(G)$

While $\neq$ null

Begin

Find support count for 
$\mathcal{W}_{x} \leftarrow \mathcal{W}_{x-1}+\frac{\mathrm{Y}_{\mathrm{x}}}{\sum_{n} \mathrm{Y}_{x}^{2}}\left(\rho^{i n, x}-\rho^{i(n-1) x}\right)$

$\rho_{s}=\left|w_{x}(t)^{x}\right|$

Assign weight for each feature

Improve new harmony search with better features

If Rule pointer $\left._{\{}\right\}$

Repeat

else

Update ruleset

endif

As a rule, wavelets are intended to give good time determination at high frequencies and good frequency determination at low frequencies. They have several favorable properties, including compact support, vanishing moments and decorrelated coefficients and have been effectively connected in signal representation and transformation. Compact support guarantees the limitation of wavelet, vanishing moment property allows wavelet focusing on most essential data and discarding noisy signal, and de-correlated coefficients property enables wavelet to reduce temporal correlation so that the correlation of wavelet coefficients are much smaller than that of the comparing temporal process. Thus, after wavelet transform, the complex signal in the time domain can be lessened into a much simpler process in the wavelet domain. By figuring the histograms of wavelet coefficients, it could get a good estimation of the probability distribution over time. The good probability estimation thus leads to a good component representation in music-mining.

\section{EXPERIMENTAL RESULTS}

\section{A. Genre Classification Accuracy}

Testing different classification algorithms for the real characterization: GMM (Gaussian Mixture Models) with three Gaussians, KNN (k-Nearest Neighbors) with $k=5$, LDA (Linear Discriminant Analysis), S1 technique and multi-class extensions of support vector machines (SVM). Support vector machines (SVM) [22] is a strategy that has indicated superb execution in binary classification issues. The classification strategy on the dataset utilized as a part of [21], which comprises of 1,000 30-second-long sound records covering ten genres with 100 documents per genre. The ten genres are Blues, Classical, Country, Disco, Hiphop, Jazz, Metal, Pop, Reggae, and Rock. The documents are gathered from radio and CD's. The experimental results are displayed in Table-1 and relating figure in Figure 11. The average accuracy of the one-versus-the-rest classifiers over a ten-fold cross validation test, are introduced in Table-2 and comparing figure in Figure 12 is very high for each of the ten classes (ranging from 91 to $99 \%)$.

Table-1 Accuracy percentage of different algorithm tested utilizing the dataset

\begin{tabular}{|c|c|c|c|c|c|c|c|}
\hline & $\begin{array}{l}\mathrm{DWCH}+\mathrm{FFT} \\
+\mathrm{MFCC}\end{array}$ & $\begin{array}{l}\text { FFT+MFCC+ } \\
\text { PITCH+BEAT }\end{array}$ & $\mathrm{FFT}+\mathrm{MFCC}$ & FFT & MFCC & PITCH & BEAT \\
\hline CMM-C & 77.4 & 74.5 & 68 & 63.1 & 57.1 & 39.8 & 29.2 \\
\hline S1 & 75.2 & 70.1 & 69.8 & 60.5 & 59 & 38.4 & 28.4 \\
\hline S2 & 78.6 & 70.9 & 70 & 61.6 & 58.6 & 35.9 & 21.2 \\
\hline MPS & 67.9 & 67.5 & 63.3 & 62.1 & 50.7 & 29.9 & 22.5 \\
\hline GMM & 63.8 & 63.6 & 59.4 & 59.5 & 49.2 & 28.1 & 22.1 \\
\hline LDA & 71.4 & 69.7 & 68.2 & 57.8 & 57.8 & 30.3 & 25.8 \\
\hline KNN & 61.2 & 60.4 & 60.9 & 52.8 & 52.1 & 22.7 & 22 \\
\hline
\end{tabular}




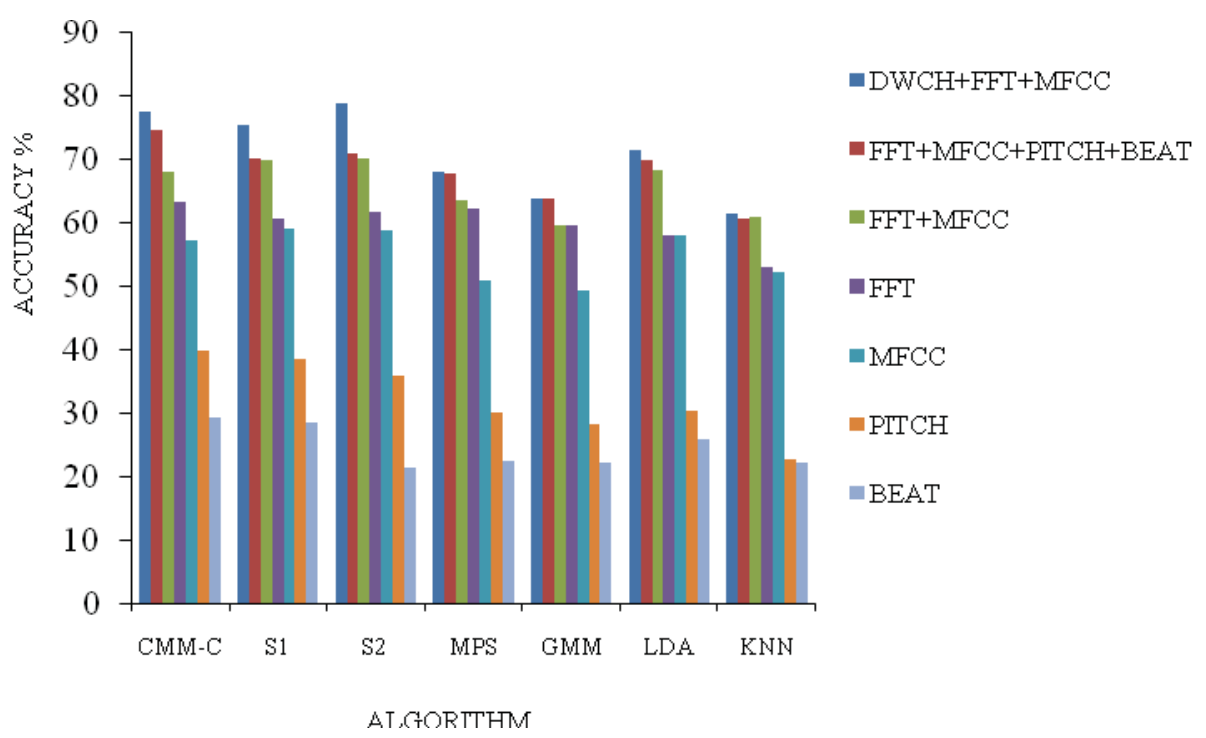

Figure 11. Graphical representation of Classification accuracy of the learning Strategies

Table-2 Accuracy percentage of S1 technique with DBCH, FFT and MFCC and Proposed Algorithm (CMM-C)

\begin{tabular}{|l|c|c|}
\hline & S1 method with DBCH, FFT and MFCC & PROPOSED ALGORITHM(CMM-C) \\
\hline BLUES & 95.049 & 96.248 \\
\hline CLASSICAL & 99.022 & 99.184 \\
\hline COUNTRY & 94.323 & 95.257 \\
\hline DISCO & 92.514 & 93.122 \\
\hline JAZZ & 98.214 & 98.049 \\
\hline METAL & 95.867 & 96.323 \\
\hline POP & 95.716 & 96.358 \\
\hline HIPHOP & 96.218 & 96.841 \\
\hline REGGAE & 92.283 & 96.077 \\
\hline ROCK & 91.658 & 92.019 \\
\hline
\end{tabular}

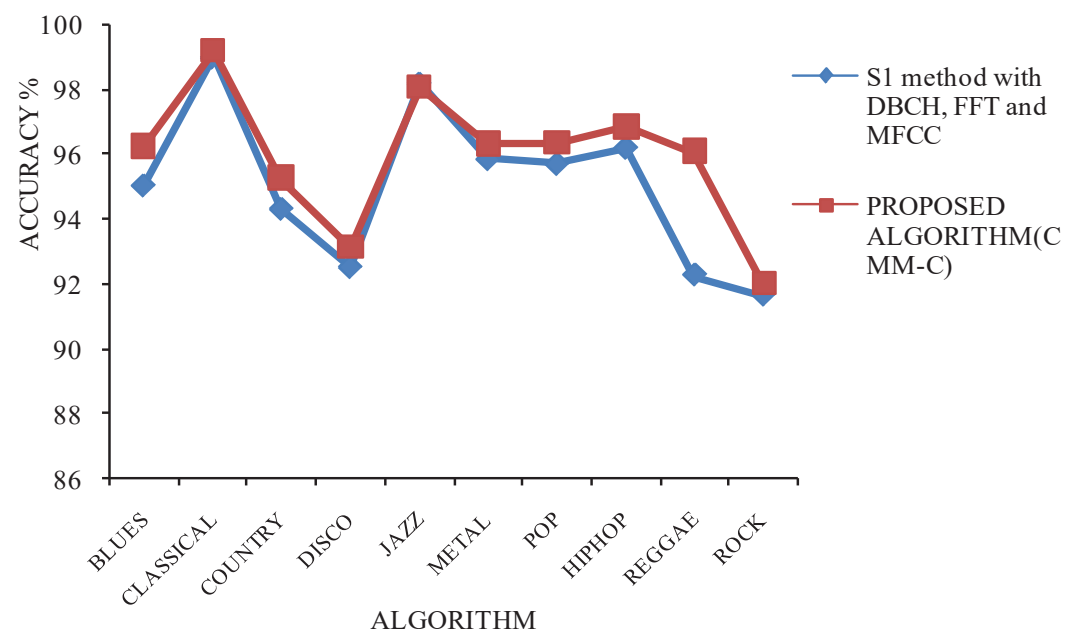

Figure 12. Comparison of S1 technique with Proposed Algorithm (CMM-C) 


\section{B. Instrumental Classification Accuracy}

The classification accuracy was tested in various composer data sets and contrasted it with numerous algorithms. The most significant gatherings are the pitch classes, octaves, note duration, and pitch gradient features. Accuracy level of the proposed collaborative music-mining classifier (CMM-C) procedure is contrasted to Numerous algorithms. The accuracy level of the elements of numerous algorithms is given in Table- 3 and comparing figure in Figure 13. The accuracy level of the elements collaborative music-mining classifier (CMM-C) is given in Table-4 and comparing figure in Figure 14.

Table-3 Accuracy Level of Numerous algorithms

\begin{tabular}{|l|l|l|l|l|}
\hline & Classical & Baroque & Classical-Keyboard & Baroque-Keyboard \\
\hline Pitch Classes & 45.1 & 33.2 & 47.2 & 42.4 \\
\hline Octaves & 67.4 & 68.6 & 72.3 & 82.6 \\
\hline Note Count & 68 & 69 & 75.6 & 83 \\
\hline Note Duration & 69.1 & 79.4 & 76.7 & 90.2 \\
\hline Pitch Gradients & 70.5 & 80.5 & 75.5 & 92 \\
\hline Pitch Range & 69.9 & 83.7 & 78.8 & 95 \\
\hline Pitch Trigram & 69.9 & 82.8 & 81 & 95.5 \\
\hline
\end{tabular}

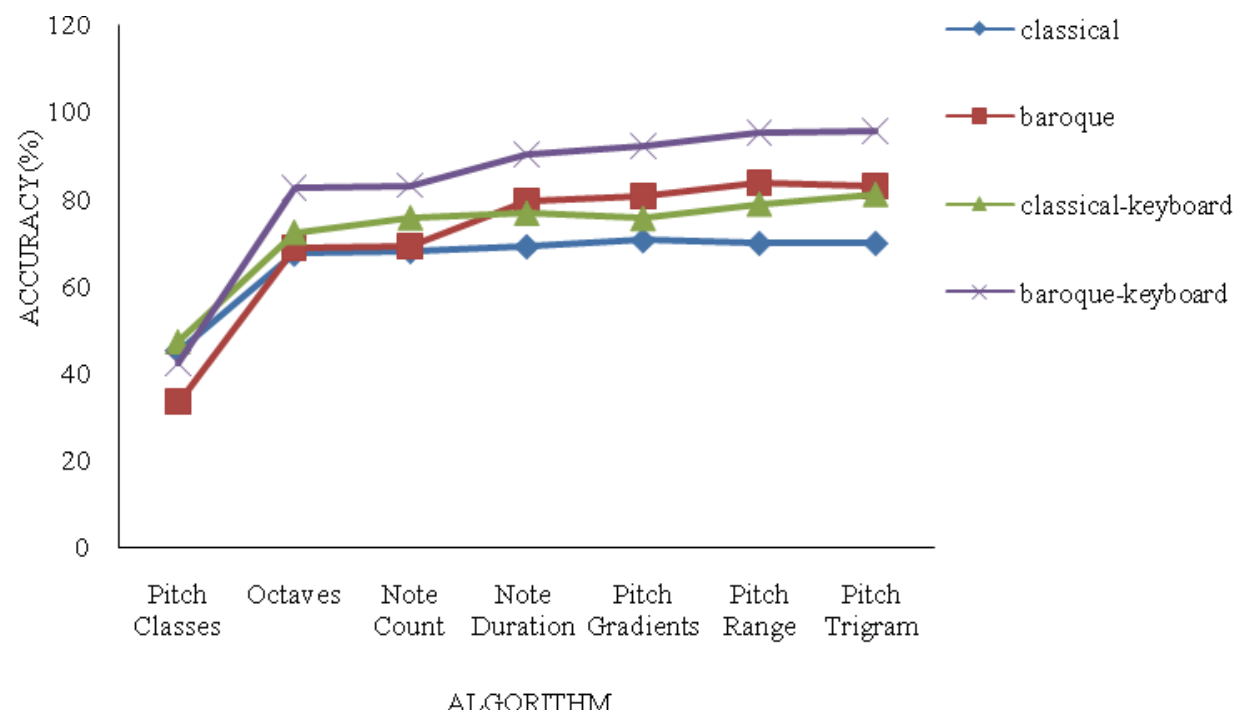

Figure 13. Graphical Representation of Accuracy Level of Numerous algorithms

Table -4 Accuracy(\%) Level of collaborative music-mining classifier (CMM-C) strategy

\begin{tabular}{|l|l|l|l|l|}
\hline & Classical & Baroque & Classical-Keyboard & Baroque-Keyboard \\
\hline Pitch Classes & 47.20 & 33.75 & 48.10 & 42.00 \\
\hline Octaves & 68.24 & 69.85 & 72.50 & 85.76 \\
\hline Note Count & 68.54 & 69.59 & 77.34 & 86.89 \\
\hline Note Duration & 73.12 & 80.62 & 78.32 & 91.10 \\
\hline Pitch Gradients & 73.46 & 82.92 & 77.11 & 92.15 \\
\hline Pitch Range & 74.87 & 83.91 & 80.02 & 96.00 \\
\hline Pitch Trigram & 74.75 & 83.00 & 81.01 & 96.50 \\
\hline Pitch Trigram & 74.75 & 83.00 & 81.01 & 96.50 \\
\hline
\end{tabular}




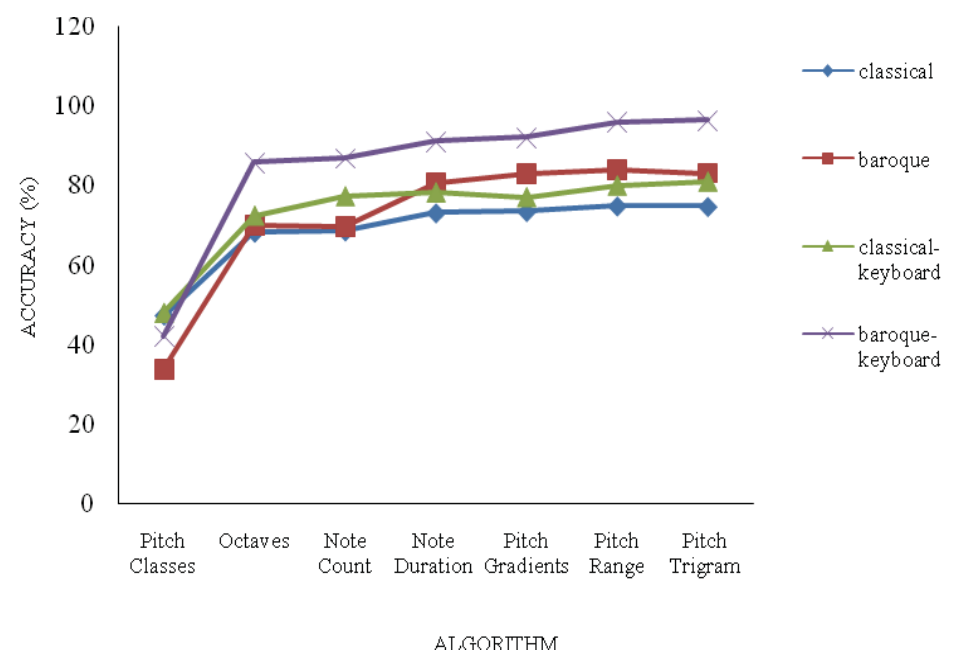

Figure14. Representation of Accuracy Level of (CMM-C) strategy

C. Overall Classification of Accuracy

The comparison of overall accuracy values of the proposed CMM-C with different algorithms is shown in Table5. Also relating graph in Figure 15

Table-5 Overall accuracy of proposed CMM-C algorithm

\begin{tabular}{|l|l|l|l|}
\hline & Instrumental & Genre & Overall \\
\hline Clustering & 47.06 & 93.02 & 70 \\
\hline MLP & 64.5 & 94.21 & 79.25 \\
\hline SVM & 81.9 & 86.76 & 84.33 \\
\hline CMM-C & 83 & 88.2 & 85.6 \\
\hline
\end{tabular}

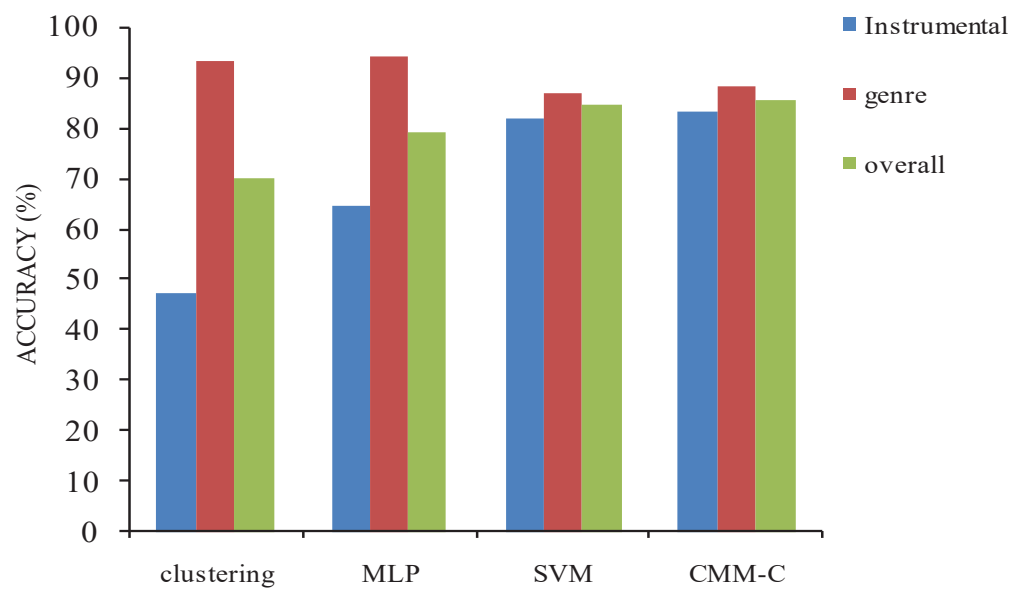

Figure 15. Representation of Overall accuracy of proposed CMM-C algorithm 


\section{CONCLUSION}

Accurate Classification of genre and instrument from music has clear advantages for the configuration of music related application or for the extraction of valuable data from huge amounts of music information. The explore displayed in this paper is an attempt to design, implement, and evaluate a genre and instrument characterization framework for a music database. The target of this work was to show a performance comparison of several classifiers, including the proposed CMM-C, KNN, LDA, GMM, MPS and SVM, for recognizing emotion from music database. The highest recognition rate is acquired with CMM-C. The overall result of this paper has demonstrated that every one of these classifiers can be utilized to perceive two dimensional space based emotions based on the 45 removed components. It additionally highlights that every classifier has their own advantages and disadvantages and combine these advantages in each classifier to achieve higher recognition rate acquires to the music-mining Algorithm. More to the point, the size of the utilized dataset is moderately small because names are difficult to acquire, especially for perceived.

\section{REFERENCES}

[1] Rajapakse, M., Wyse, L., "Generic audio classification using a hybrid model based on gmms and hmms", In: IEEEP, pp.1550-1555, 2005.

[2] Lidy, T., Rauber, A, "Evaluation of feature extractors and psycho-acoustic transformations for music genre classification", In: ISMIR, 2005.

[3] W.Walker, P.Lamere, P.Kwok,B.Raj, E.Gouvea, P. Wolf, and J. Woelfel, "Sphinx-4: A flexible open source framework for speech recognition", cmus phinx.sourceforge.net/sphinx4/doc/Sphinx4White paper.pdf, 2004.

[4] J. T. Foote, "Content-based retrieval of music and audio", in SPIE, pp. 138-147.

[5] B. Logan and S. Chu, "Music summarization using key phrases", in IEEE Conf. on Acoustics, Speech and Signal Processing, 2000.

[6] B. Logan, "Mel frequency cepstral co-effcients for music modelling", in Intl. Symposium on Music Information Retrieval, 2000.

[7] L. R. Rabiner and B. H. Juang, "Fundamentals of Speech Recoognition Prectice-Hall”, 1993.

[8] Beth Logan, "Mel Frequency Cepstral Coefficients for Music Modeling", In Proceedings of International Symposium on Music Information Retrieval (ISMIR), 2000.

[9] George Tzanetakis and Perry Cook, "Musical genre classification of audio signals", IEEE Transactions on Speech and Audio Processing, Vol.10, No.5, 2002.

[10] Tao Li and Mitsunori Ogihara, "Detecting emotion in music", In Proceedings of the Fifth International Symposium on Music Information Retrieval (ISMIR2003), pp.239-240, 2003.

[11] Tao Li and Mitsunori Ogihara, "Content-based music similarity search and emotion detection", In Proceedings of the 2004 IEEE International Conference on Acoustics, Speech, and Signal Processing, pp.V705- V708, 2004.

[12] Tao Li and Mitsunori Ogihara, "Music artist style identification by semisupervised learning from both lyrics and content", In Proceedings of the ACM Conference on Multimedia, pp. 364-367, 2004.

[13] Tao Li, Mitsunori Ogihara, and Qi Li, “A comparative study on content based music genre classification”, In Proceedings of 26th Annual ACM Conference on Research and Development in Information Retrieval (SIGIR 2003), pp.282-289, 2003.

[14] Tao Li and George Tzanetakis, "Factors in automatic musical genre classification of audio signals", In Proceedings of 2003 IEEE Workshop on Applications of Signal Processing to Audio and Acoustics (WASPAA'03), pp.143-146, 2003.

[15] Schindler, Alexander, and Andreas Rauber. "An Audio-Visual Approach to Music Genre Classification through Affective Color Features", Advances in Information Retrieval. Springer International Publishing, pp.61-67, 2015.

[16] Y. Liu, X. Li, R. Te, C. Pan, X. Zang, "Extracting music genes for era classification", Exp. Syst. Appl, Vol. 41, pp.5520-5525, 2014.

[17] P. Dhanalakshmi, S. Palanivel, V. Ramalingam., "Classification of audio signals using AANN and GMM", Appl. Soft Comput.. Vol.11, pp.716-723, 2011.

[18] P. Dhanalakshmi, S. Palanivel, V. Ramalingam. "Pattern classification models for classifying and indexing audio signals", Eng. Appl. Artif. Intell, Vol.24, pp.350$357,2011$.

[19] Gary Weiss and Foster Provost, "The effect of class distribution on classifier learning: An empirical study", Technical Report ML-TR 44, Rutgers University, 2001.

[20] Bianca Zadrozny and Charles Elkan, "Learning and making decisions when costs and probabilities are both unknown", In Proceedings of the Seventh ACM SIGKDD International Conference on Knowledge discovery and data mining (SIGKDD 2001), pp.204-213, 2001.

[21] Tzanetakis and Cook, Tzanetakis, G. and Cook, P, "Musical genre classification of audio signals, IEEE Transactions on Speech and Audio Processing”, Vol. 10, No.5, 2002.

[22] Vapnik, V. N., "Statistical learning theory", John Wiley \& Sons, New York, 1998. 